Proc. Indian Acad. Sci. (Chem. Sci.), Vol. 105, No. 6, December 1993, pp. 695-708.

(C) Printed in India.

\title{
First photothermal deflection spectroscopy set-up in Egypt
}

\author{
SOHAIR NEGM \\ Prof. Hassan Talaat Laboratory, Advanced Laser Spectroscopy Laboratory, Ain Shams \\ University, Abbassia, Cairo, Egypt \\ Present address: Faculty of Engineering (Shoubra), Zagazig University (Benha), Zagazig, \\ Egypt
}

\begin{abstract}
We have succeeded in carrying out some ultra-sensitive photothermal experiments, using photothermal deflection spectroscopy (PDS) (or mirage effect). We have also employed the PDS technique to study the optical and thermal properties of solid/air interfaces. The experimental set-up of the transverse PDS has been described.

The experimental resuits show that there is an exponential decay in PD signals with increase in the chopper frequency as well as with an increase of the phase difference. The PD signal decreases with increasing the probe beam normal offset to allow us to calculate the thermal diffusion length of the deflection medium (air in our case). We determined the thermal diffusion of air to be $=0.43 \mathrm{~mm}$ at the chopper frequency $50 \mathrm{~Hz}$, which is in agreement with published values. Further, we employed the PD technique to obtain the spectra for two dyes [Rhodamine 6G (Rh6G) and Crystal Violet (CV)] of different optical absorption structures. The PDS shows a good resolution of the peaks of $\mathrm{CV}$ which are hardly separated in optical absorption measurements.

Finally, a comparison of the photoacoustic spectra (PAS) of the two dyes was carried out, which showed that PDS has better spatial spectral resolution.
\end{abstract}

Keywords. Photothermal deflection spectroscopy; properties of solid/air interfaces; optical absorption.

\section{Introduction}

Absorption spectroscopy is normally carried out indirectly through measurement of the incident, reflected and/or transmitted intensities, from which one can determine the absorbed intensity. Among the principal techniques to measure absorption directly are the photothermal deflection (PD) and photoacoustic (PA) spectroscopies. Both spectroscopies measure the heat deposited in the material due to the absorption of photons, either optically in the case of PD or acoustically in the case of PA. It is well known that upon the absorption of electromagnetic radiation by a given medium, a fraction or all the excitation energy is converted into thermal energy through de-excitation processes. This de-excitation mechanism has provided the physical basis for the highly sensitive PD spectroscopy. This technique was first introduced in 1980 by Boccara et al (1980) and subsequently developed by Aamodt and Murphy (1981) and by Jackson et al (1981). PD spectroscopy is a powerful and novel experimental method in which the absorption coefficient of the sample is deduced from the measurements of the thermal gradient in the gas layer adjacent to the sample surface. 
In this method, the sample is illuminated with a chopped monochromatic light "pump beam" and, when the sample absorbs any of the energy incident on it, some of the energy levels in the sample are excited and subsequently these energy levels must be de-excited. The most common mode of de-excitation is through the non-radiative or heating mode and thus the optical excitation of the sample results in a periodic heat flow from the sample to the surrounding media. This, in turn, results in a corresponding change in the index of refraction. By probing this variation of the refractive index by a second weak "probe beam", it experiences a periodic deflection synchronous with the intensity modulation. The amplitude and phase of the periodic deflection of the probe beam can be measured with a position sensor. This deflection is directly related to the optical absorption of the sample as will be shown in the next section. The positioning of the probe beam with respect to the pump beam allows for two choices in performing PDS: collinear configuration, where the gradient of the index of refraction is both created and probed within the sample, and transverse configuration, in which the index of refraction is accomplished in a thin layer adjacent to the sample surface. The latter is the case that will be discussed in this work. The photothermal technique has many advantages:

(1) The technique could be employed for samples whose optical absorptions are difficult to obtain directly, e.g. powdered samples, as also for samples that are completely opaque to transmitted light.

(2) It also incorporates the thermal properties of the sample and its surroundings, hence, this property, like thermal diffusivity and conductivity, could be determined.

(3) It is an ultra-high sensitive method for probing the thermal properties of matter. The temperature rise associated with this process is of the order of $10^{5}-10^{4} \mathrm{C}$. The technique is easily used for absorption detection.

(4) It surmounts the limitation of PA (where the sample has to be enclosed in a PA cell) and measurements may often be made in open sample configuration with substantial reduction in experimental complexity.

(5) Acoustic shielding requirements are less stringent.

(6) There is also no background due to window absorption.

(7) PD works in hostile environments such as at extreme temperatures or in caustic substances.

Because of the above advantages, for the use of PDS and our experience in PAS, it was decided to construct a set-up for PDS (according to Fournier and Boccara) (Boccara et al 1980) measurements in air, in an on-going project to study materials whose optical absorptions are normally difficult to obtain.

In this paper, we present our initial results using the PDS set-up to determine the optical absorption of dye lasers in powder form and to compare the obtained spectra with those obtained using PAS.

\section{Theory}

In order to correlate the deflection signal to processes occurring at the sample surface, it is necessary to determine: (1) The temperature distribution $T$ in the sample and the surrounding media as a function of position and time; (2) the optical beam propagation through the non-homogeneous medium; (3) the relation between the deflection and the output voltage of the position sensor. 


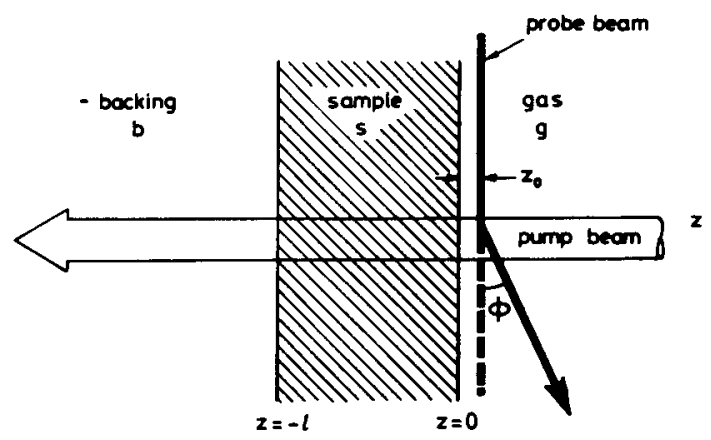

Figure 1. Geometry of the thermal field evaluation (transverse PDS).

\subsection{Temperature distribution in the sample and the surrounding media}

Consider the geometry as shown in figure 1; the sample is placed on a backing material and surrounded by a fluid (air in our case). The boundary planes are asssumed to be parallel and infinitely extended in the radial direction. The sample is illuminated perpendicularly by a modulated laser beam or a lamp as we have used in our work. Assume that both the gas and the backing material are optically non-absorbing media. The absorbed radiation heats the sample and diffuses through the gas-sample and the backing material. The time dependent a.c. component of the temperature $T$ satisfies the equations (Salazar et al 1989),

$$
\begin{aligned}
& \nabla^{2} T_{\mathrm{g}}-\left(1 / \kappa_{\mathrm{g}}\right)\left(\partial T_{\mathrm{g}} / \partial t\right)=0, \text { for gas, } \\
& \nabla^{2} T_{\mathrm{s}}-\left(1 / \kappa_{\mathrm{s}}\right)\left(\partial T_{\mathrm{s}} / \partial t\right)=-\left(Q(r, t) / k_{\mathrm{s}}\right), \text { for sample, } \\
& \nabla^{2} T_{\mathrm{b}}-\left(1 / \kappa_{\mathrm{b}}\right)\left(\partial T_{\mathrm{b}} / \partial t\right)=0, \text { for backing, }
\end{aligned}
$$

subject to the following boundary condition.

(i) Temperature continuity at the sample surface:

$$
T_{\mathrm{g}} \mathrm{l}_{z=0}=T_{\mathrm{s}} \mathrm{l}_{z=0} \& T_{\mathrm{s}} \mathrm{l}_{z=-l}=T_{\mathrm{b}} \mathrm{l}_{z=-l}
$$

(ii) Heat flow continuity at the sample surface:

$$
\left.k_{\mathrm{g}} \frac{\partial T_{\mathrm{g}}}{\partial z}\right|_{z=0}=\left.\left.k_{\mathrm{s}} \frac{\partial T_{\mathrm{s}}}{\partial z}\right|_{z=0} \& k_{\mathrm{s}} \frac{\partial T_{\mathrm{s}}}{\partial z}\right|_{z=-l}=\left.k_{\mathrm{b}} \frac{\partial T_{\mathrm{b}}}{\partial z}\right|_{z=-l} ;
$$

where $l$ is the sample thickness, $k_{(i)}$ is the thermal conductivity of the material (i) $\left(\mathrm{cal} / \mathrm{cm} \mathrm{s}^{\circ} \mathrm{C}\right), \rho_{(i)}$ the density of the material $(i)\left(\mathrm{g} / \mathrm{cm}^{3}\right), c_{(i)}$ the specific heat of the material (i) (cal $/ \mathrm{g}^{\circ} \mathrm{C}$ ), and $\kappa_{(i)}=k_{(i)} / \rho_{(i)} \cdot c_{(i)}$, the thermal diffusivity of material $(i)$. (i) can have subscripts $\mathbf{s}, \mathrm{g}, \mathrm{b}$, representing sample, gas and backing material (Rossencwaig and Gersho 1976). $Q(r, t)$ is the heat deposited per unit volume oscillating with frequency $\omega$ and can be given by:

$$
Q(r, t)=\left(P_{0} \alpha / \pi a^{2}\right) \exp (\alpha z) \exp \left(-r^{2} / \mathrm{a}^{2}\right) \frac{1}{2} \exp (i \omega t),
$$

where $P_{0}$ is the power of the exciting beam, $\alpha$ is the optical absorption coefficient of the sample and $a$ is the beam radius defined at $1 / e$ of the intensity. 
The set of equations (1), with initial conditions (2), has been solved using the method of separation of variables in cylindrical geometry. The solutions are

$$
\begin{aligned}
& T_{\mathrm{g}}(r, z, t)=\frac{1}{2} \int_{0}^{\infty} \delta J_{0}(\delta r) E(\delta) \exp \left(-\beta_{g} z\right) \exp (i \omega t) \mathrm{d} \delta, \\
& T_{\mathrm{s}}(r, z, t)=\frac{1}{2} \int_{0}^{\infty} \delta \mathrm{d} \delta J_{0}(\delta r)\left[\Gamma(\delta) \exp (\alpha z)+A(\delta) \exp \left(\beta_{\mathrm{s}} z\right)\right. \\
& \left.+B(\delta) \exp \left(-\beta_{\mathrm{s}} z\right)\right] \exp (i \omega t) \\
& T_{\mathrm{b}}(r, z, t)=\frac{1}{2} \int_{0}^{\infty} \delta J_{0}(\delta r) D(\delta) \exp \left(\beta_{\mathrm{b}}(l+z)\right) \exp (i \omega t) \mathrm{d} \delta,
\end{aligned}
$$

where $\beta_{i}^{2}=\delta^{2}+\left(i \omega / \kappa_{i}\right)(i=\mathrm{g}, \mathrm{s}, \mathrm{b})$ is the relation between the separation constants, and $J_{0}$ is the Bessel function of zero order and we have:

$$
\begin{aligned}
& A(\delta)=-[\Gamma(\delta) / H(\delta)]\left[(1-\mathrm{g})(\mathrm{b}-P) \exp (-\alpha l)+(1+\mathrm{b})(\mathrm{g}+P) \exp \left(\beta_{\mathrm{s}} l\right)\right] \\
& B(\delta)=-[\Gamma(\delta) / H(\delta)][(1+\mathrm{g})(\mathrm{b}-P) \exp (-\alpha l) \\
& \left.+(1-b)(\mathrm{g}-P) \exp \left(-\beta_{\mathrm{s}} l\right)\right] \\
& D(\delta)=\Gamma(\delta) \exp (-\alpha l)+A(\delta) \exp \left(-\beta_{\mathrm{s}} l\right)+B(\delta) \exp \left(\beta_{\mathrm{s}} l\right), \\
& E(\delta)=\Gamma(\delta)+A(\delta)+B(\delta), \\
& H(\delta)=(1+\mathrm{g})(1+\mathrm{b}) \exp \left(\beta_{\mathrm{s}} l\right)-(1-\mathrm{g})(1-\mathrm{b}) \exp \left(-\beta_{\mathrm{s}} l\right) . \\
& g=\left(k_{\mathrm{g}} \beta_{\mathrm{g}} / k_{\mathrm{s}} \beta_{\mathrm{s}}\right) \text {, } \\
& b=\left(k_{\mathrm{b}} \beta_{\mathrm{b}} / k_{\mathrm{s}} \beta_{\mathrm{s}}\right) \text {, } \\
& P=\left(\alpha / \beta_{\mathrm{s}}\right) \text {. }
\end{aligned}
$$

The physical interpretation of (4), (5) and (6) is that any temperature distribution can be decomposed into distribution of the form of

$$
J_{0}(\delta r) \exp \left(-\beta_{i} z\right)
$$

The distributions act independently of each other and have an effective thermal length given by

$$
l_{i}=\left(1 / \mathscr{R} e\left(\beta_{i}\right)\right)=\left[\mathscr{R e}\left(K_{i}^{2}+\delta^{2}\right)^{1 / 2}\right]^{-1} .
$$

The case of $\delta=0$ and $i \omega / \kappa$ can be expanded to real and imaginary parts. Then $\beta=(1+i)\left(\omega / 2 \kappa_{i}\right)^{1 / 2}$, which is the one-dimensional solution given by Rossencwaig and Gersho (1976). $\delta$ can then be expressed as a parameter to show how the heat is diffused in directions other than the $z$-direction.

\subsection{Temperature distribution for transverse PDS}

For transverse PDS, the probe beam propagates completely within the gas. The material is divided into two classes according to the optical absorption coefficient (Salazar et al 1989) 
2.1a Optically opaque solids ( $\alpha$ l $\gg 1)$ : In these materials the optical length $(\mu \alpha=1 / \alpha)$ is much smaller than the sample thickness $l$ and (4) $T_{8}$ can be expressed as:

$$
\begin{array}{r}
T_{\mathrm{g}}(r, z)=\frac{1}{2} \frac{P_{0}}{2 \pi k_{s}} \int_{0}^{\infty} \delta J_{0}(\delta r) \exp \left(-\delta^{2} a^{2} / 4\right) \frac{1}{\beta_{\mathrm{s}}}\left(\frac{1+\exp \left(-2 \beta_{\mathrm{s}} I\right)}{1-\exp \left(-2 \beta_{\mathrm{g}} D\right.}\right) \\
\exp \left(-\beta_{\mathrm{g}} z\right) \mathrm{d} \delta
\end{array}
$$

2.2b Optically transparent solids $(\alpha l \ll 1)$ : The optical absorption length of these materials is much greater than the sample thickness and the temperature distribution in the gas is given by (Salazar et al 1989)

$$
T_{\mathrm{z}}(r, z)=\frac{1}{2} \frac{P_{0}}{2 \pi k_{\mathrm{s}}} \int_{0}^{\infty} \delta J_{0}(\delta r) \exp \left(-\delta^{2} a^{2} / 4\right) \frac{\alpha}{\beta_{\mathrm{s}}^{2}} \exp \left(-\beta_{\mathrm{g}} z\right) \mathrm{d} \delta
$$

\subsection{Optical beam deflection for transverse PDS}

In the case of transverse PDS, the probe beam is directed parallel to and grazing the sample surface through the gas and is deflected by an amount (Salazar et al 1989)

$$
\phi=(1 / n)(\mathrm{d} n / \mathrm{d} T) \int_{-\infty}^{\infty} \nabla_{\perp} T_{\varepsilon} \times \mathrm{d} S,
$$

where $n$ is the gas refractive index and $S$ is the ray path, see figure 2 where the $x$-axis is the probe beam direction. There are two components of the probe beam deflection: the transverse component $\phi_{t}$ and the normal component $\phi_{n}$, where

$$
\begin{aligned}
& \phi_{\mathrm{t}}=-(1 / n)(\mathrm{d} n / \mathrm{d} T) \int_{-\infty}^{\infty}\left(\partial T_{\mathrm{g}} / \partial y\right) \mathrm{d} x \hat{K}, \\
& \phi_{n}=(1 / n)(\mathrm{d} n / \mathrm{d} T) \int_{-\infty}^{\infty}\left(\partial T_{\mathrm{g}} / \partial z\right) \mathrm{d} x \hat{J}
\end{aligned}
$$

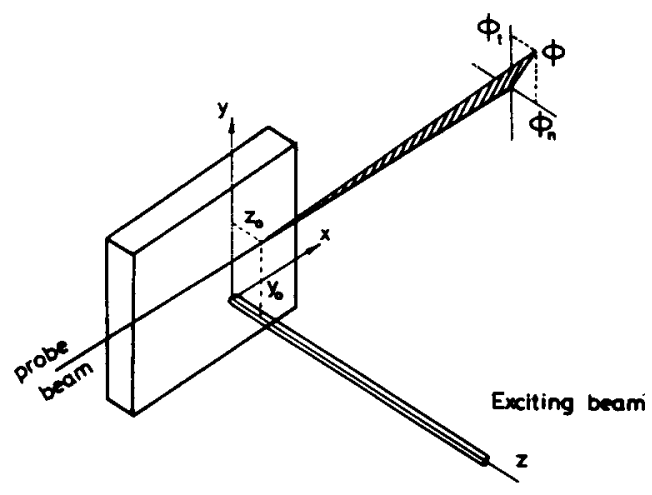

Figure 2. Geometry for the "mirage" deflection. The probe beam has a transverse offset $y_{0}$ and a normal offset $z_{0}$. 
Using (4) for $T_{\mathrm{g}}$ and taking into consideration the integral transform relation (Murphy and Aamodt 1980) one can write:

$$
\begin{aligned}
& \phi_{\imath}=(1 / n)(\mathrm{d} n / \mathrm{d} T) \int_{0}^{\infty} \delta \sin (\delta y) E(\delta) \exp \left(-\beta_{\mathrm{g}} z\right) \exp (i \omega t) \mathrm{d} \delta \hat{K} \\
& \phi_{n}=(1 / n)(\mathrm{d} n / \mathrm{d} T) \int_{0}^{\infty} \beta_{\mathrm{g}} \cos (\delta y) E(\delta) \exp \left(-\beta_{\mathrm{g}} z\right) \exp (i \omega t) \mathrm{d} \delta \hat{J}
\end{aligned}
$$

and by classifying the material into two parts we get the following relations.

2.3a For optically opaque solids:

$$
\begin{aligned}
\phi_{t}= & -\frac{1}{n} \frac{\mathrm{d} n}{\mathrm{~d} T} \frac{P_{0}}{2 \pi k_{\mathrm{s}}} \exp (i \omega t) \int_{0}^{\infty} \delta \sin (\delta y) \exp \left(-\delta^{2} a^{2} / 4\right) \\
& \times \frac{1}{\beta_{\mathrm{s}}}\left(\frac{1+\exp \left(-2 \beta_{\mathrm{s}} l\right)}{1-\exp \left(-2 \beta_{\mathrm{s}} l\right.}\right) \exp \left(-\beta_{\mathrm{g}} z\right) \mathrm{d} \delta \hat{K}, \\
\phi_{n}= & \frac{1}{n \mathrm{~d} n} \frac{P_{0}}{2 \pi k_{\mathrm{s}}} \exp (i \omega t) \int_{0}^{\infty} \beta_{\mathrm{g}} \cos (\delta y) \exp \left(-\delta^{2} a^{2} / 4\right) \\
& \times \frac{1}{\beta_{\mathrm{s}}}\left(\frac{1+\exp \left(-2 \beta_{\mathrm{s}} l\right)}{1-\exp \left(-2 \beta_{\mathrm{s}} l\right)}\right) \exp \left(-\beta_{\mathrm{g}} z\right) \mathrm{d} \delta \hat{J} .
\end{aligned}
$$

2.3b For optically transparent solids:

$$
\begin{aligned}
& \phi_{t}=-\frac{1}{n} \frac{\mathrm{d} n}{\mathrm{~d} T} \frac{P_{0}}{2 \pi k_{\mathrm{s}}} \exp (i \omega t) \int_{0}^{\infty} \delta \sin (\delta y) \exp \left(-\delta^{2} a^{2} / 4\right) \frac{\alpha}{\beta_{\mathrm{s}}} \exp \left(-\beta_{\mathrm{g}} z\right) \mathrm{d} \delta \hat{K} \\
& \phi_{n}=\frac{1}{n} \frac{\mathrm{d} n}{\mathrm{~d} T} \frac{P_{0}}{2 \pi k_{\mathrm{s}}} \exp (i \omega t) \int_{0}^{\infty} \beta_{\mathrm{g}} \cos (\delta y) \exp \left(-\delta^{2} a^{2} / 4\right) \frac{\alpha}{\beta_{s}} \exp \left(-\beta_{\mathrm{g}} z\right) \mathrm{d} \delta \hat{J}
\end{aligned}
$$

where $P_{0}$ is the pump beam power, $z$ is the normal offset, $y$ is the transverse offset. The above two equations show that the deflection is proportional to the power of the pump beam and exponentially decays with the modulation frequency.

\subsection{The relation between the deflection and the output voltage of the position sensor}

The deflection of the beam due to the change in the index of refraction of the medium adjacent to the sample is detected by the position sensor with converts the deflection into an output voltage. The change of the signal $\Delta V$ above the d.c. level is calculated as shown in figure 3. Assuming a Gaussian probe beam

$$
\frac{\Delta V}{V}=\frac{\Delta I}{I_{0}}=4 \Delta x \int_{0}^{\infty} \frac{2}{\pi \omega_{2}^{2}} \exp \left(-2 r^{2} / \omega_{2}^{2}\right) \mathrm{d} r=\frac{4}{(2 \pi)^{1 / 2}} \frac{\Delta x}{\omega_{2}},
$$

since $\phi d=\Delta x$, where $d$ is the distance from the focal spot of the probe beam to the 


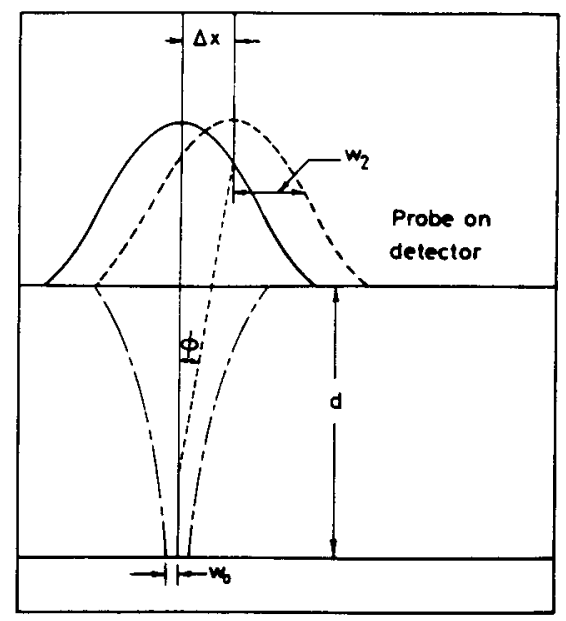

Figure 3. Probe beam on the detector.

detector, $I_{0}$ is the probe beam intensity, and $\omega_{2}$ is the radius of the spot on the detector.

$$
\omega_{2}=\lambda d /\left(\pi \omega_{0} n_{0}\right)
$$

where $\omega_{0}$ is the probe beam radius at the focal spot. Hence,

$$
\Delta V=\left[4 /(2 \pi)^{1 / 2}\right] \phi\left(\pi \omega_{0} n_{0} / \lambda\right) V .
$$

This equation shows that the output signal is independent of the distance between the sample and the detector for modulation frequency of the order of few hundred cycles per second.

\section{Experimental procedure}

We have carried out the transverse (PD) technique (Jackson et al 1981), in our work here. The set-up is shown in figure 4. A $1000 \mathrm{~W}$ tungsten-halogen lamp is used, with its housing cooled by water circulation and small air fan. The light from the lamp is focussed by a glass lens $L_{1}$ into the entrance slit of the monochromator (Carl Zeiss Jena. 287804) to provide a tunable light source. A mechanical chopper (variable speed) $[15 \mathrm{~Hz}-10 \mathrm{kHz}$ light chopper, Noise Interference Type Boston Electronic (617) $566-382]$ is located in front of the entrance slit of the monochromator. Its purpose is to chop (interrupt) the light beam and to provide the reference signal for detection. The monochromator is motorized to allow for accurate scan of the wavelength. The output light was focussed on the sample by using camera lens $\mathbf{L}_{2}$. An $\mathrm{He}-\mathrm{Ne}$ laser (high pointing stability $0.5 \mathrm{~mW}$ laser uniphase 1101 power supply Model 1201 (408) $434-1800$ ) with output wavelength $6328 \AA$ served as the optical probe beam. This beam is focussed by a convex lens $L_{3}$ with focal length $10 \mathrm{~cm}$. The deflection of the probe was monitored by a position sensor (split photo diode) detector low noise preamplifier Ithaco Model 1202 (607) 272-7640 at $60 \mathrm{~cm}$ from the sample. The output of the position sensor amplifier was fed into a synchro-He ${ }^{+}$lock-in amplifier (Princeton Applied Research Model 186A) (Murphy and Aamodt 1980). 


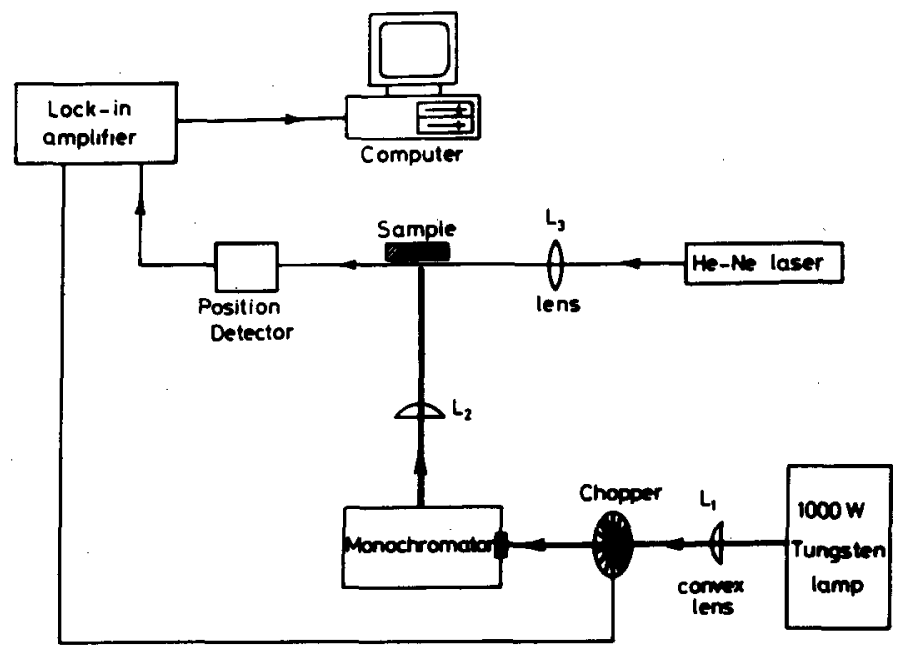

Figure 4. Experimental set-up of the transverse (PDS) technique.

The square-wave output signal generated by the chopper is applied to the reference signal input of the lock-in amplifier. The output from the lock-in amplifier is connected to an $x-y$ recorder or a personal computer. In order to isolate the set-up from possible laboratory vibrations that can prove to have a very destructive effect on the PDS signal, a specially designed sand box was used to avoid these vibrations. The PDS components were also held to a heavy iron plate that was placed on the sand box. This design was very effective in reducing possible vibrations leading to a noisy signal. Under these conditions, maximum deflection and good transverse resolution across the heated region were achieved.

\section{Results and discussions}

4.1 The dependence of the amplitude and the phase of the signal on the chopper frequency at constant value of the normal offset $z_{0}$

To check the correspondence of the change of the signal amplitude and phase with the chopper frequency, and to see how it follows the theory, the dependence of the amplitude and the phase of the signal were determined as the frequency of the chopper was changed (at constant value of normal offset $z_{0}$ ). The experimental results of PD signal of the carbon black sample versus the chopper frequency $(\omega)$ are shown in figure 5. The plots show that the PD signal amplitude falls off as $1 / \omega$. This is in agreement with theory, (22), and the literature (Jackson et al 1981). This result was repeated for three values of the probe beam offset $z_{0}$. Further; by comparing these results for the three normal offset values of $z_{0}$, one can see that the signal amplitude decreases as the probe beam offset increases. From both these observations, one can conclude that there is a strong dependence of the signal on the modulated frequency and also on the probe beam offset $z_{0}$ as indicated by theory (Aamodt and Murphy 1981). Since the main source of the PD signal is the sample surface temperature, each component (spatial frequency) has different effective thermal diffusion length. The high spatial terms contribute to the rapidly varying temperature profile, while the low 


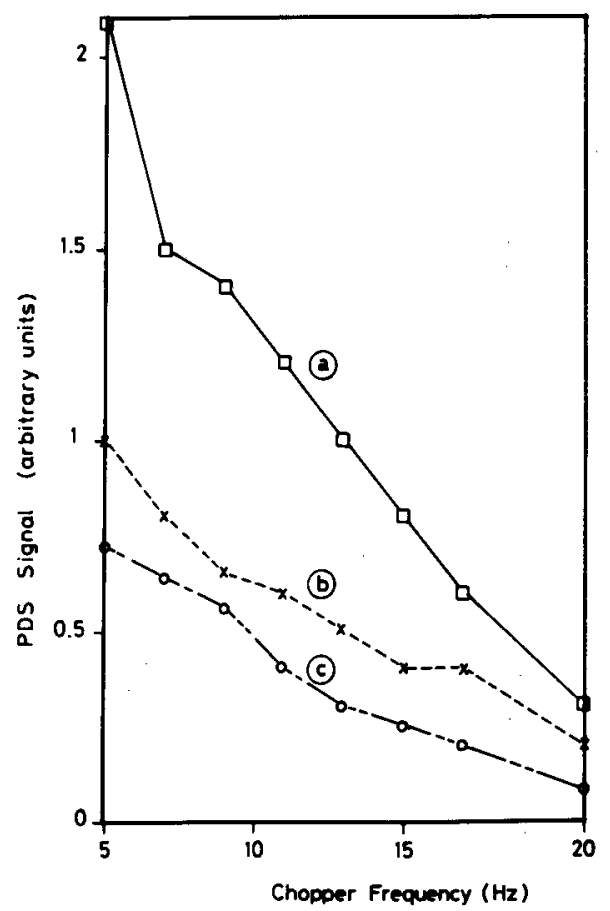

Figure 5. The transverse PDS vs. the chopper frequency at probe beam normal offset. $z_{0}=250 \mu \mathrm{m}$ (a), $500 \mu \mathrm{m}$ (b), $750 \mu \mathrm{m}$ (c).

spatial frequency terms determine its slowly varying coarser feature. Since the thermal diffusion length is a measure of how heat diffusing from a heat source can travel and remain coherent with the excitation source modulation, and since each spectral component has its own effective thermal diffusion length, the sample volume contributing to the surface temperature will be different for each component. Since $\mu_{i}=\left(2 k_{i} / \omega\right)^{1 / 2}$ where $\mu_{i}$ is the thermal diffusion length of material $i$, for high modulation frequency the thermal diffusion length of the sample will be small and the contribution comes only from a small volume. On the other hand, for low spatial frequency, $\mu_{\mathrm{s}}$ is larger and the contribution comes from a big volume, thus the coarser features are determined. Figure 6 shows the increase in the phase difference of the PDS signal as the chopping frequency increases. The phase difference is a function of the diffusion length of the sample, and it indicates that there is a time delay between the excitation of the sample and subsequent deflection of the beam.

4.2 The dependence of the signal on the normal offset $z_{0}$ at constant modulation frequency

In this part, as can be seen from figure 7 , the effect on the PDS signal of varying the value of the normal probe beam offset $z_{0}$. In this case again, the sample was standard Carbon Black sample. There is an exponential increase of the signal as the probe beam approaches the sample surface. The exponential dependence which is illustrated in (22a) where $\phi \propto \exp \left(-z_{0} / l_{\mathrm{g}}\right)$ and $l_{g}=\left(2 k_{\mathrm{g}} / \omega\right)^{1 / 2}$. We can see that there is also a strong dependence of the PDS signal on the probe beam offset $z_{0}$. The exponential 


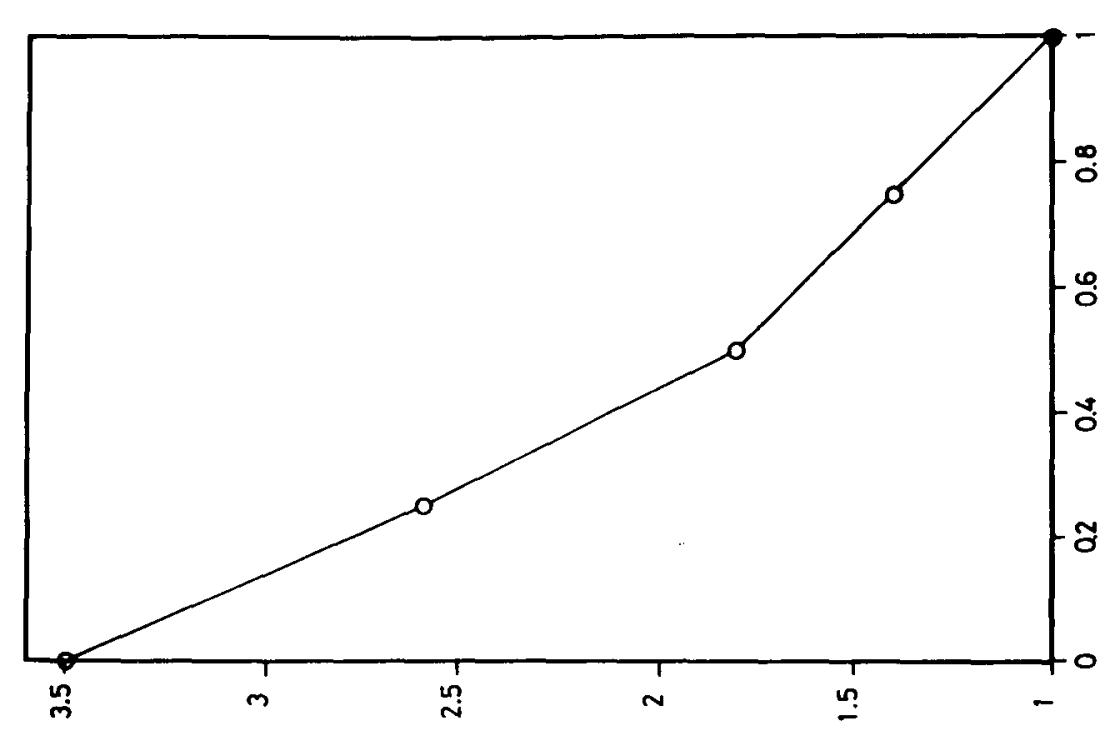

(5!!un KJDJ!ado) joub!s sod

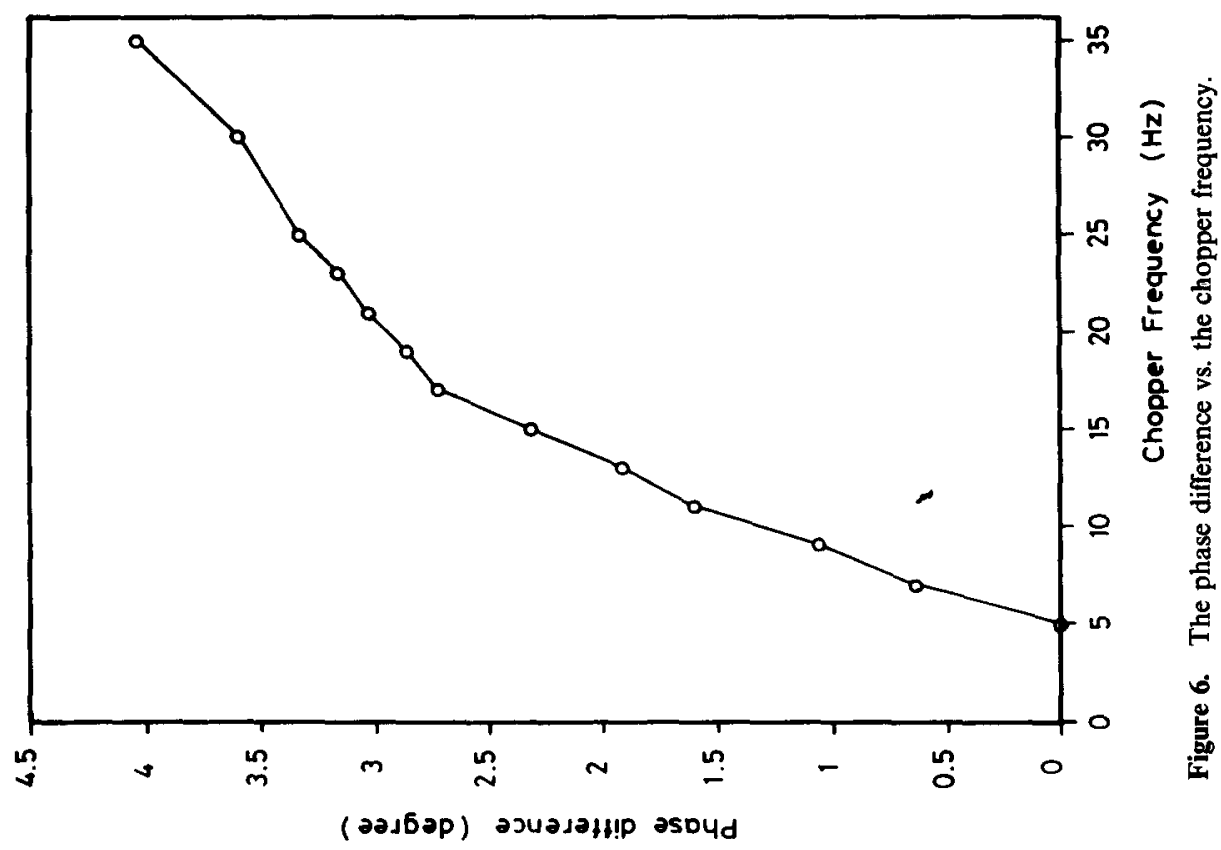


decrease of the signal with the increasing values of $z_{0}$ can be used to determine the diffusivity of the deflecting medium (air). By taking the logarithm of both sides of $\left(\phi \propto \exp \left(-z_{0} / t_{g}\right)\right.$ we get

$$
\ln \phi=k-\left(\omega / 2 k_{\mathrm{g}}\right)^{1 / 2} z_{0},
$$

which is a straight line whose slope is $\left(\omega / 2 k_{\mathrm{g}}\right)^{1 / 2}$; this gives the value of the thermal diffusion length $\left(l_{\mathrm{g}}\right)$ of the air to be $\approx 0.438 \mathrm{~mm}$ at $50 \mathrm{~Hz}$. This is in agreement with the published value given by Boccara et al (1983).

\subsection{Spectral results}

We also employed the PD technique to investigate photothermal signal resolution of the absorption bands of two dyes with differing absorption bands. These dyes are: Rhodamine 6G (Rh6G) and Crystal Violet (CV) which has two overlapping bands at $550 \mathrm{~nm}$ and $580 \mathrm{~nm}$ which could hardly be separated optically. The normalized PD spectra of the two dyes are shown in figure 8. One can observe from the figures that the PDS spectrum for Rh6G exhibits a peak at $\approx 530 \mathrm{~nm}$ while in the case of $\mathrm{CV}$ it shows a peak at $\approx 560 \mathrm{~nm}$.

As mentioned earlier, the transverse PDS has spatial resolution on the sample surface. We have measured three spectra for Crystal Violet dye (CV) at different values of the probe beam normal offset $z_{0}$. These spectra are shown in figure 9. It can be seen from the figures that for large values of $z_{0}$ the spectrum is wide and
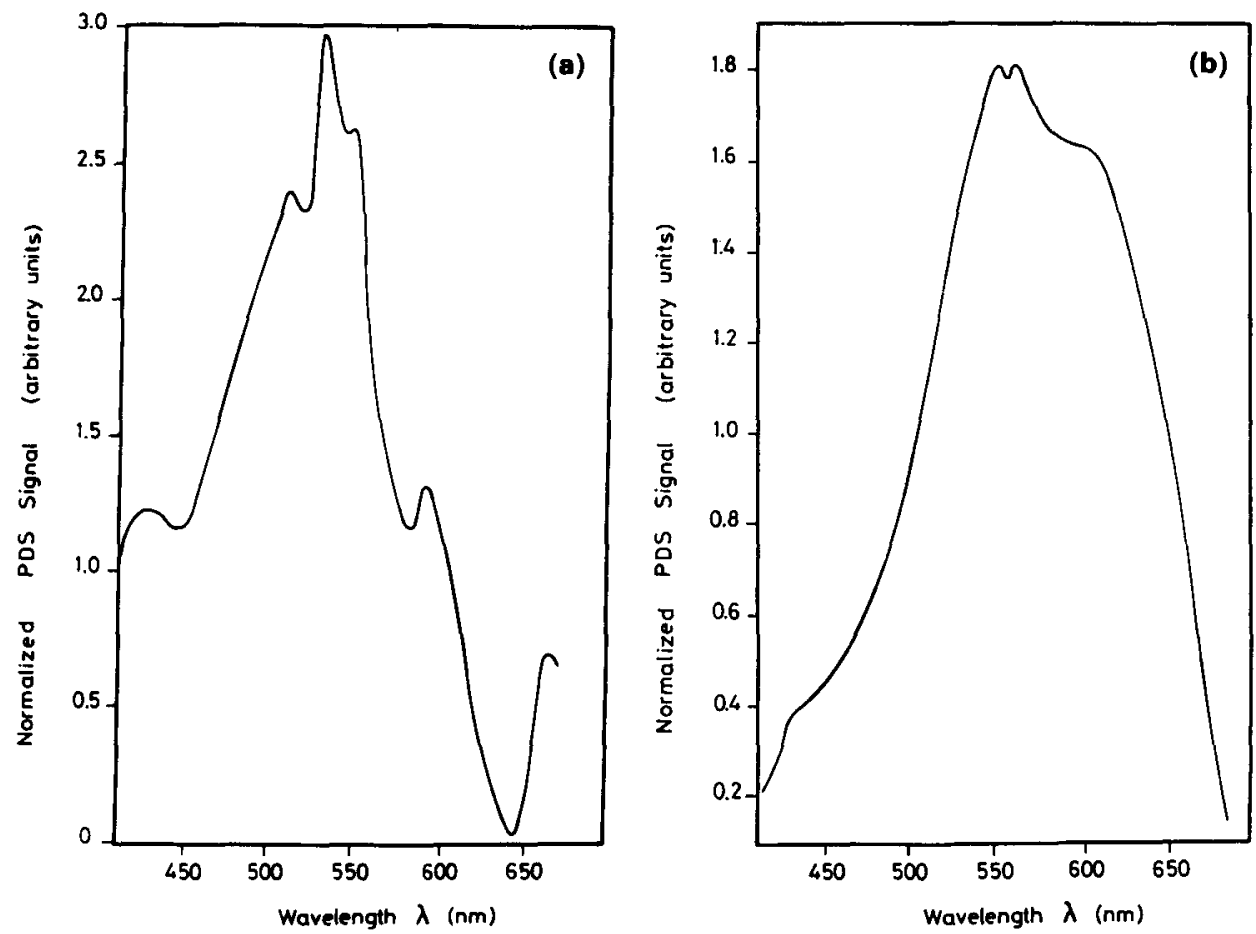

Figure 8. The normalized PD spectra of $\operatorname{Rh} 6 \mathrm{G}$ (a) and $\mathrm{CV}$ (as powder) (b) at chopper frequency $14 \mathrm{~Hz}$. 

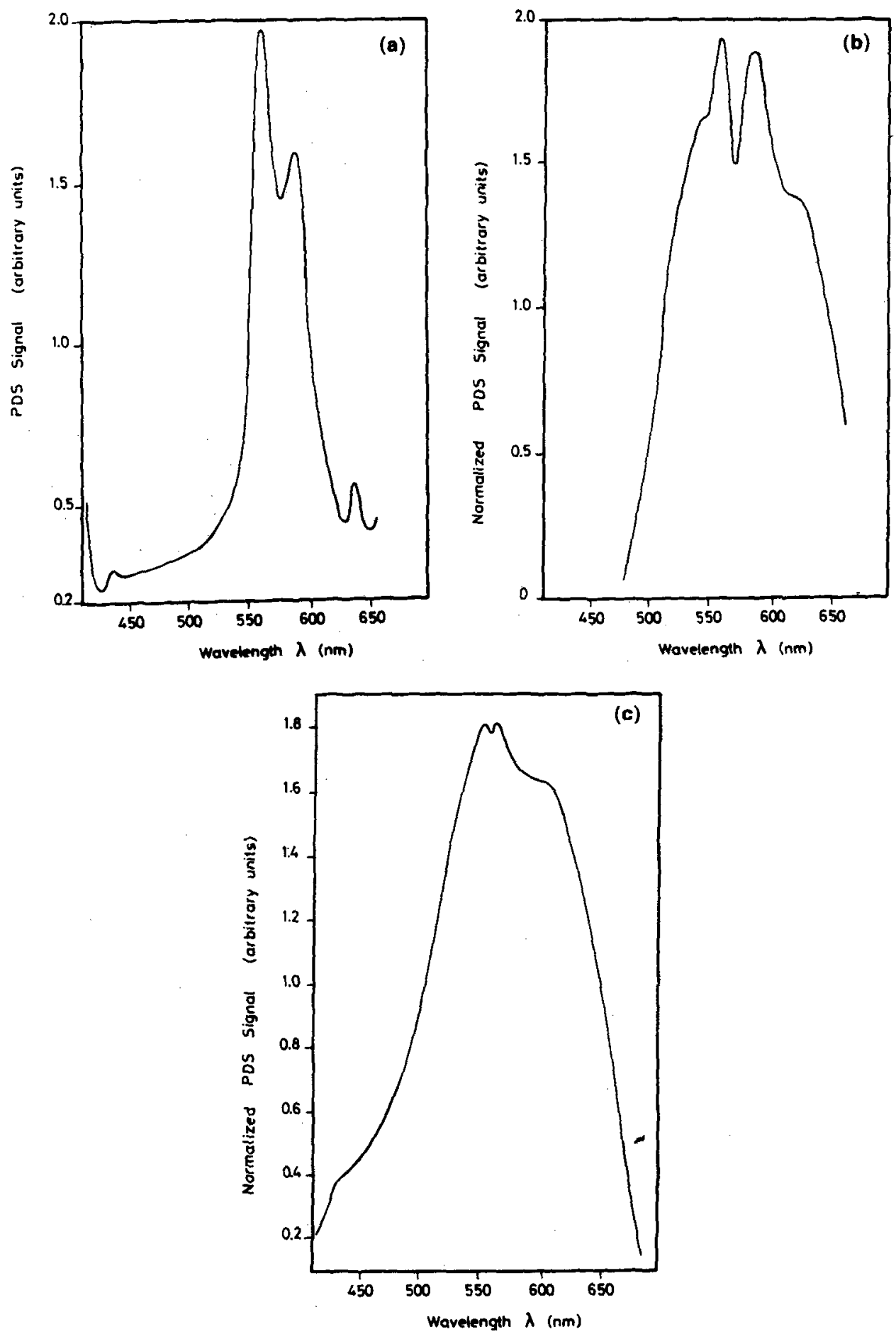

Figure 9. The normalized spectra of $\mathrm{CV}$ at chopper frequency $14 \mathrm{~Hz}$. The probe beam normal offset: $z_{0}=85 \mu \mathrm{m}$ (a), $125 \mu \mathrm{m}$ (b), $175 \mu \mathrm{m}$ (c). 


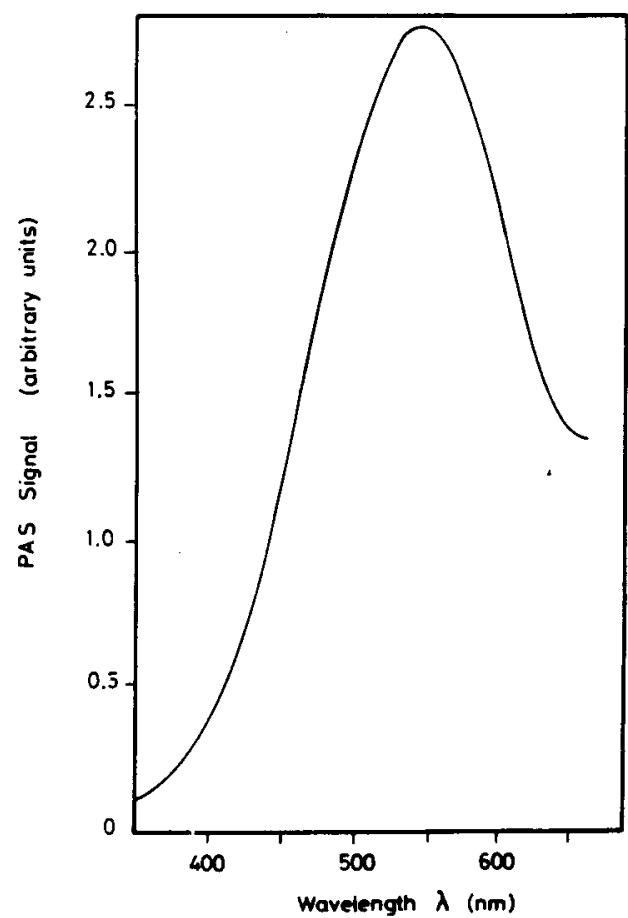

Figure 10. Normalized PAS of Rh6G (solid) at chopper frequency $14 \mathrm{~Hz}$.

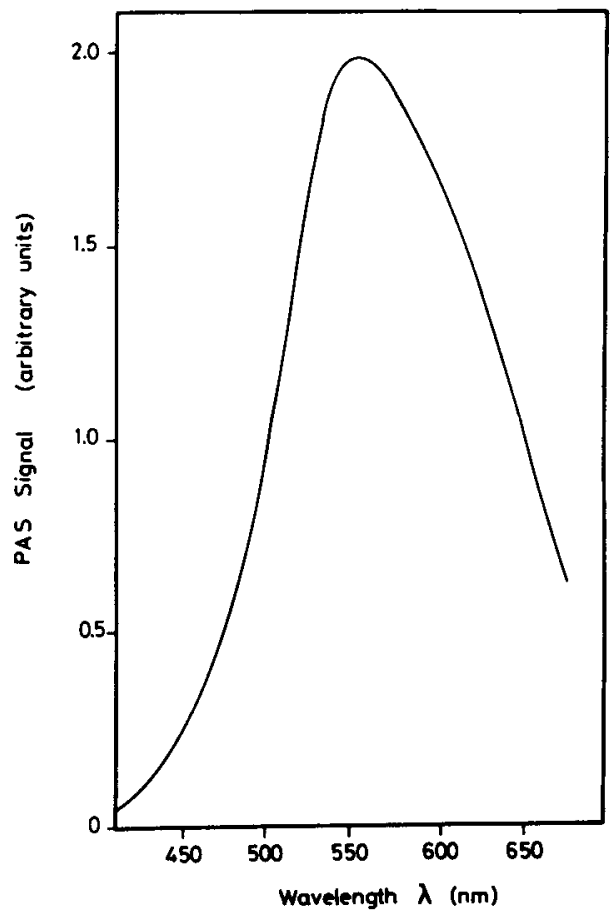

Figure 11. Normalized PAS of CV (solid) at chopper frequency $14 \mathrm{~Hz}$.

smooth and there is a complete masking of the much sharper peaks of $\mathrm{CV}$. This is because at large values of $z_{0}\left(z_{0}=175 \mu \mathrm{m}\right)$, the observed spectrum is a collection of the average values of the contributions of all points of the inhomogeneous sample surface. For the smaller value of $z_{0}$ we can see that the spectrum is much narrower and noiser. The two peaks, seen at wavelengths 550 and $580 \mathrm{~nm}$, became regular. For the smallest value of $z_{0}$ used $(85 \mu \mathrm{m})$, the two peaks become very narrow and are well-resolved. This shows that the PDS has much better resolution than regular optical absorption. Using PDS we are able to resolve the two peaks that normally overlap.

\subsection{Comparison with photoacoustic spectroscopy}

Since PDS and PA detection provide similar experimental information, a comparison of these two methods is of interest. We compared our experimental PD spectra with the PA spectra for the same two dye materials (Rh6G and CV). The figures 10, 11 show the normalized PA spectra of the two dyes. We observe from these figures and from figure 8 that the PA spectra of both Rh6G and CV are broader and form smoother bands than that of the corresponding PD spectra even at large values of the probe beam offset $z_{0}$. 


\section{Conclusion}

As we have seen, we have succeeded in designing, building and calibrating a complete set-up for a newly developed spectroscopic technique in our laboratory in Egypt, namely Transverse Photothermal Deflection Spectroscopy. Though the technique seemed at times during development to be hard, difficult and prone to noise caused by vibration, particularly at the point of detection, the ease of application of the technique to the study of both the thermal and optical properties for solid interfaces finally justified such efforts.

We have also carried out a comparative study of the PDS and the photoacoustic PA spectra for the two dyes (Rh6G and CV) under the same conditions. The PD spectra were recorded at different normal offset distances from the samples and then compared to the PA spectra which showed that PDS was able to resolve the two peaks of $\mathrm{CV}$, which are hardly resolved by either optical or PA measurements. The PDS proved to be superior to the PA due to its higher capability in spatial and spectral resolutions. These are initial results and we plan future work along these lines.

\section{Acknowledgement}

I would like to express my sincere gratitude to my professor Dr. Hassan Talaat for his guidance, encouragement and enriching discussions throughout this work.

\section{References}

Aamodt L C and Murphy J C 1981 J. Appl. Phys. 524903

Boccara A C, Fournier D and Bodz J 1980 Appl. Phys. Lett. 36130

Jackson W B, Amer N A, Boccara A C and Fournier D 1981 Appl. Opt. 201333

Murphy J C and Aamodt L C $1980 \mathrm{~J}$. Appl. Phys. 514580

Rossencwaig A and Gersho A 1976 J. Appl. Phys. 4764

Salazar A, Sanchez A -L and Fernandez J 1989 J. Appl. Phys. 654150 